OPEN ACCESS

Tariq, F., Nix, AR., \& Love, D. (2000). Efficient implementation of pilotaided 32 QAM for fixed wireless and mobile ISDN applications. 680 684. https://doi.org/10.1109/VETECS.2000.851545

Peer reviewed version

Link to published version (if available):

10.1109/VETECS.2000.851545

Link to publication record in Explore Bristol Research

PDF-document

University of Bristol - Explore Bristol Research

General rights

This document is made available in accordance with publisher policies. Please cite only the published version using the reference above. Full terms of use are available:

http://www.bristol.ac.uk/red/research-policy/pure/user-guides/ebr-terms/ 


\title{
EFFICIENT IMPLEMENTATION OF PILOT-AIDED 32 QAM FOR FIXED WIRELESS AND MOBILE ISDN APPLICATIONS
}

\author{
M. Fahim Tariq, Andrew Nix \& Derek Love ${ }^{\dagger}$ \\ Centre for Communications Research \\ University of Bristol \\ Merchant Venturers Bldg., Woodland Rd. \\ Bristol BS8 IUB, United Kingdom \\ M.F.Tariq@bristol.ac.uk, Andy.Nix@bristol.ac.uk \\ ${ }^{\dagger}$ Linear Modulation Technology \\ Intek Global House \\ Westfield Industrial Estate, Midsomer Norton, \\ Bath, BA3 4BS, United Kingdom \\ Derek.Love@linearmod.com
}

\begin{abstract}
This paper presents the design and performance of a pilot tone-aided 32 QAM modem developed for wireless ISDN applications. The modem is capable of transmitting a gross data rate of $70 \mathrm{kbit} / \mathrm{s}$ in a $20 \mathrm{kHz}$ channel spacing. A low complexity receiver has been designed that allows the effects of fading and frequency offset to be removed digitally at a low IF using information extracted from the pilot tone. The design has been fully evaluated in narrowband Rayleigh and Rician fading channels. Computer simulations show that extreme Doppler shifts and frequency offsets have little effect on system performance. The design is suitable for mobile radio as well as fixed applications such as Wireless in the Local Loop (WLL).
\end{abstract}

\section{INTRODUCTION}

The growing demand for basic rate ISDN services (64 $\mathrm{kbit} / \mathrm{s}$ ) over the PSTN has jeopardised the future of many wireless networks. The prime aim of every wireless operator is to match the services offered over wired networks. This has lead researchers all over the world to investigate novel ways of squeezing more and more data into band limited radio channels. Among the techniques used to increase throughput, one of the most common solutions is the use of higher level linear modulation schemes that transmit multiple bits per symbol.

When comparing modulation schemes, the QAM family can be shown to offer an excellent compromise between bits per second per hertz and symbol error performance in a noisy channel [1]. However, the very nature of the wireless environment results in gross amplitude and phase distortion that must be accurately compensated in order to successfully receive data using high levels of QAM. Extensive research dating back to the early 1980's produced two famous methods for compensating multiplicative distortion in a narrowband wireless channel. These methods rely on the transmission of a reference signal along with the data. The reference is used in the receiver to estimate the precise nature of the channel's amplitude and phase distortion. The first technique inserts pilot symbols in the information sequence [2], whereas the second technique transmits a continuous pilot tone together with the data spectrum to assist the demodulation process [3].

The modem presented in this paper is based on the Pilot Tone Aided (PTA) concept. A gap is created in the centre of the information bearing signal spectrum and a pilot tone inserted prior to transmission. At the receiver, the tone is extracted from the received signal and used for the purpose of channel estimation and compensation. PTA can be considered as the frequency dual of Pilot Symbol Aided (PSA) correction. However, both systems have important practical differences [4].

The PTA concept was introduced in 1979 with the first mobile voice implementation published by $\mathrm{McGeehan}$ and Bateman [3] in 1984. Promising field trail results together with cheap and widely available DSP devices paved the way for the PTA concept to be applied to data transmission. In the late $1980 \mathrm{~s}$, Martin and Bateman [5][6] implemented a 16 QAM PTA data transmission system for operation in multipath fading channels. The proposed system was referred to as Transparent Tone In Band (TTIB) and was capable of transmitting up to 9.6 $\mathrm{kbit} / \mathrm{s}$ using the V.29 modem standard in a $2.7 \mathrm{kHz}$ channel bandwidth.

The system developed in this paper makes use of the 32 cross QAM constellation. The system achieves a gross data rate of $70 \mathrm{kbit} / \mathrm{s}$ in a $20 \mathrm{kHz}$ channel bandwidth. The data rate is in excess of the basic ISDN rate $(64 \mathrm{kbit} / \mathrm{s})$ and additional coding or control information could also be sent. A low complexity digital receive architecture is used to accurately estimate and then correct for channel distortion. This method also allows frequency offsets introduced by local oscillator drift to be inherently corrected without the need for additional hardware. A novel low IF approach is used in the receiver to avoid the need for Hilbert transforms in the data extraction procedure. These transforms add significant ripple in the pass-band and while this is acceptable for QPSK, the degradation is too severe for a 32 QAM constellation. This new receive method differs substantially from the conventional approach originally proposed by Martin, Bateman and McGeehan.

\section{SYSTEM DESCRIPTION}

Figure 1 shows the block diagram for the transmitter. A pilot tone is inserted in the centre of the transmit spectrum by splitting the incoming data into two parallel subbands. Each subband operates at $35 \mathrm{kbit} / \mathrm{s}$ with data mapped onto separate 32 cross QAM constellations. This results in a gross $14 \mathrm{kbaud} / \mathrm{s}$ (7kbaud/s per subband). 


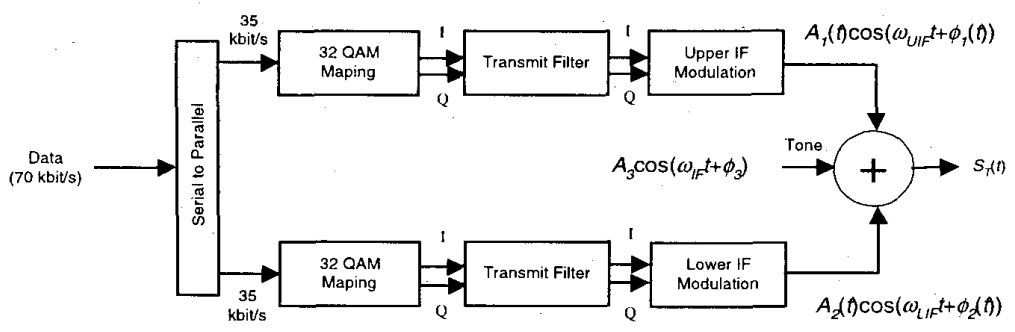

Figure 1: Block diagram for the 32 QAM PTA transmitter

The two subbands are modulated onto an upper intermediate frequency (UIF) and a lower intermediate frequency (LIF) with a gap remaining for the required tone insertion. The gap bandwidth depends on the maximum frequency offset and Doppler shift to be corrected in the system. In this particular system, a gap bandwidth of $700 \mathrm{~Hz}$ has been used at a centre frequency of $14 \mathrm{kHz}$. This choice results in a relaxed specification for the bandlimiting root raised cosine filters with an excess bandwidth factor of 0.38 . The low IF signal at the transmitter can be written mathematically as:

$$
\begin{aligned}
S_{T}(t)= & A_{I}(t) \cos \left(\omega_{U / F} t+\phi_{I}(t)\right)+A_{2}(t) \cos \left(\omega_{L I F} t+\phi_{2}(t)\right)+ \\
& A_{3} \cos \left(\omega_{I F} t+\phi_{3}\right)
\end{aligned}
$$

where $A_{l}(t), A_{2}(t), A_{3}$ represent the amplitudes, $\omega_{\text {tIF }}, \omega_{\text {LIF }}$, $\omega_{1 F}$ the frequencies and $\phi_{l}(t), \phi_{2}(t), \phi_{3}$ the phases of the upper intermediate subband, lower intermediate subband and pilot tone respectively.

When the signal arrives at the receiver, it is corrupted by amplitude and phase distortion as well as Additive White Gaussian Noise (AWGN). The IF signal at the receiver can be written as:

$$
\begin{aligned}
S^{\prime}{ }_{R}(t)= & A_{1}(t) A_{c h}(t) \cos \left(\omega_{\text {IIF }} t+\phi_{t}(t)+\phi_{c h}(t)\right)+ \\
& A_{2}(t) A_{c h}(t) \cos \left(\omega_{\text {LIF }} t+\phi_{2}(t)+\phi_{c h}(t)\right)+ \\
& A_{3} A_{c h}(t) \cos \left(\omega_{i F} t+\phi_{3}+\phi_{c h}(t)\right)+n(t)
\end{aligned}
$$

where $A_{c h}(t)$ and $\phi_{c h}(t)$ represent the amplitude and phase of the distortion introduced by the radio channel and $n(t)$ represents the contribution due to AWGN.

In communication systems, when the received signal is down converted from RF to IF, it is common for local oscillator drift to cause frequency offset in the received signal. This makes the received signal impossible to detect. Equation 2 can be modified to incorporate the effect of frequency drift as shown below:

$$
\begin{aligned}
S_{R}(t)= & A_{l}(t) A_{c h}(t) \cos \left(\omega_{V I F} t+\omega_{D} t+\phi_{I}(t)+\phi_{c h}(t)\right)+ \\
& A_{2}(t) A_{c h}(t) \cos \left(\omega_{L I F} t+\omega_{D} t+\phi_{2}(t)+\phi_{c h}(t)\right)+ \\
& A_{3} A_{c h}(t) \cos \left(\omega_{h F} t+\omega_{D} t+\phi_{3}+\phi_{c h}(t)\right)+n(t)
\end{aligned}
$$

where $\omega_{D}$ represents the frequency drift (in radians) introduced by the oscillators. In this design, two-stages of frequency correction are used. A coarse frequency correction stage is implemented at IF to correct gross frequency errors. After this process, a maximum frequency error of $\pm 150 \mathrm{~Hz}$ remains. A digital baseband fine frequency correction procedure has been simulated in this paper to correct this residual error.

Demodulation and detection of the received signal requires accurate compensation for the channel's amplitude and phase distortion. Figure 2 shows the block diagram for the 32 QAM PTA receiver. The pilot tone is extracted by digitally filtering the received signal spectrum at baseband. The channel estimation and compensation block is used to estimate the amplitude and phase distortion from the extracted tone. This information is used to generate pre-distorted upper and lower subband carriers. Each carrier is multiplied with the received signal to automatically translate the subbands to baseband while correcting for local oscillator drifts and radio channel distortion. The baseband signals contain images that are removed by passing them through low pass root raised cosine (RRC) filters before sending the samples for hard decision and 32 QAM demapping. The two $35 \mathrm{kbit} / \mathrm{s}$ data streams are finally combined to form the original binary signal.

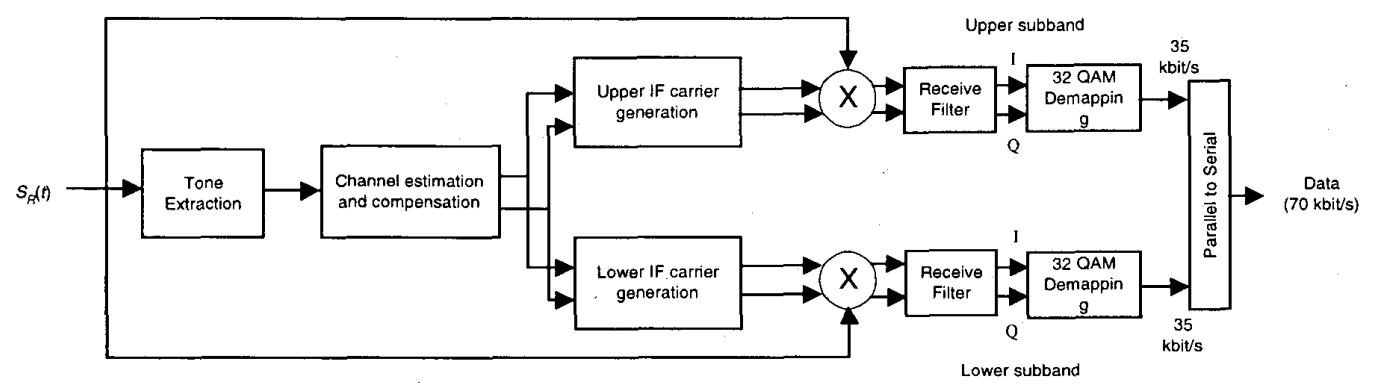

Figure 2: Block diagram of 32 QAM PTA receiver 


\section{PILOT TONE TO DATA POWER}

In the generation of $S_{T}(t)$, the ratio of the pilot tone power to the data power plays an important role in defining the overall performance of the system. At the receiver, if the pilot tone is buried in noise, it produces a noisy reference that degrades system performance considerably. On the other hand, if the pilot tone power is very high, it reduces the power in the data bands and this makes it difficult for the 32-QAM constellation to be detected. The pilot tone to data power ratio is defined mathematically as:

$$
R=2 A_{3}{ }^{2} /\left(E\left[A_{1}{ }^{2}(t)\right]+E\left[A_{2}{ }^{2}(t)\right]\right)
$$

where $E[$.$] represents the mean or expected value.$

In this study, the pilot tone to data ratio has been optimised by performing a simulation in AWGN for the proposed 32-QAM modem. The results are presented in Figures $3 \mathrm{a} \& 3 \mathrm{~b}$ and show that $R=0.2$ minimises the error rate and hence this value is used in all subsequent simulations and system evaluations.

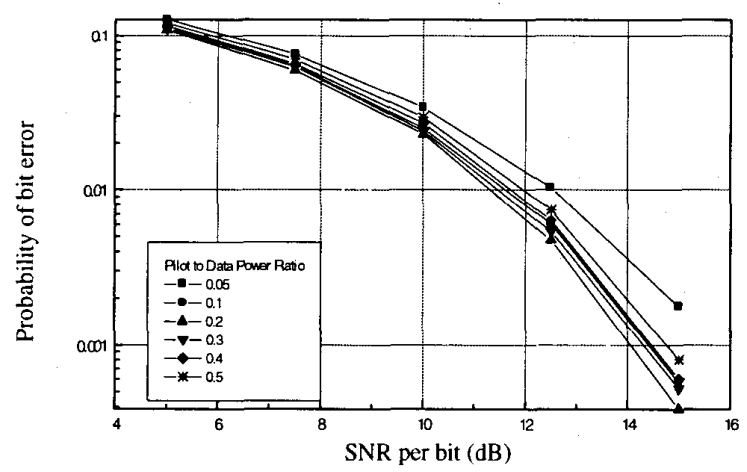

Figure 3a: BER of 32-QAM in AWGN for different values of pilot to data power ratio

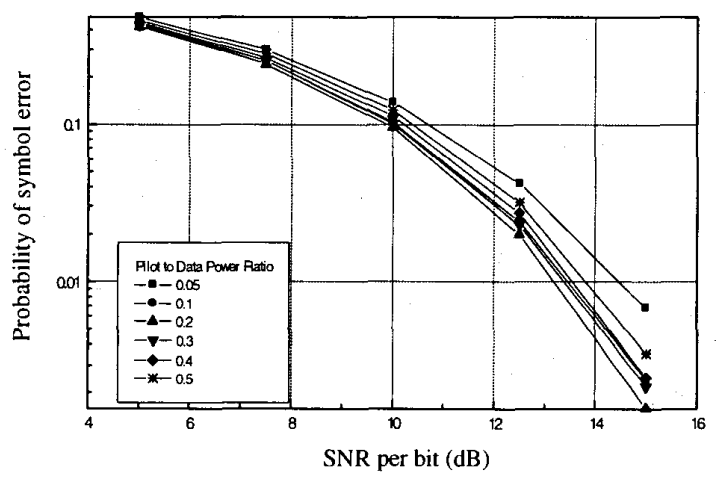

Figure 3b: SER of 32-QAM in AWGN for different values of pilot to data power ratio

\section{PEAK TO MEAN POWER}

High pilot tone powers also result in a larger peak-tomean power ratio at the transmitter and this translates into greater amplifier linearity requirements and/or backoff levels. The peak-to-mean power ratio for the transmitted signal is defined mathematically as:

$$
\eta=\frac{\max \left\{S_{T}(t)\right\}}{E\left[S_{T}(t)\right]}
$$

A simulation study has been performed to calculate the ratio $\eta$ by drawing the cumulative probability for the transmit peak-to-mean power ratio under linear power steps of 0.05 Watts. Figure 4 indicates that the peak-tomean transmit power is exponentially distributed. The cumulative graph also indicates that for $99.9 \%$ of the time the peak-to-mean power ratio remains below a linear value of 8.4 (relative to the mean power). Converting this value into the more usual $\log$ form, the $99.9 \%$ peak-tomean value is found to be $9.24 \mathrm{~dB}$. Signal powers above this value should be limited in the transmitter.

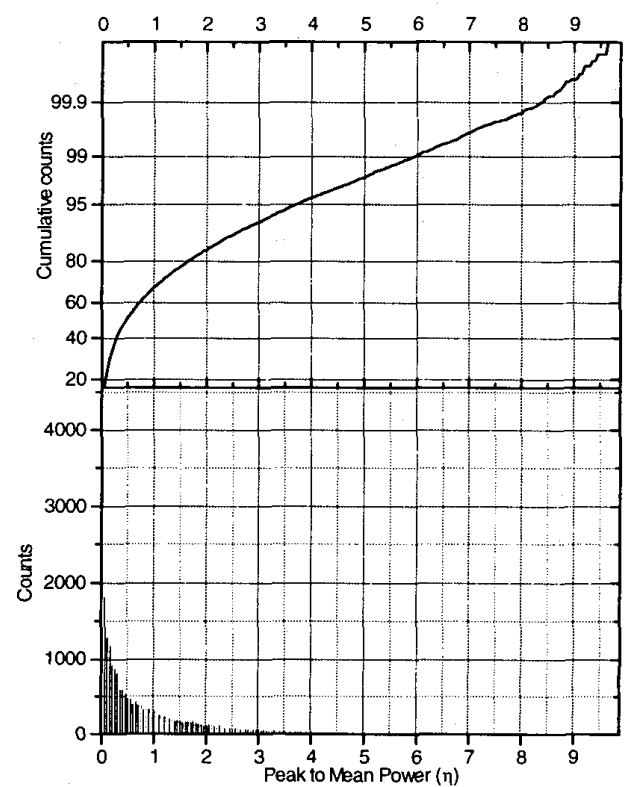

Figure 4: Histogram and cumulative count of peak-tomean transmitted power

\section{RAYLEIGH PERFORMANCE}

The performance of the system in a Rayleigh fading environment has been evalutated for a range of different Doppler spreads and local oscillator frequency offsets. To generate time correlated Rayleigh fading, an 8-ray model defined by [7] has been used. The In-phase and Quadrature components of this model are defined mathematically as:

$$
\begin{aligned}
& I_{c h}(t)=\sum_{n=1}^{8} A_{n} \cos \left(\theta_{n}+2 \pi f_{d} t \cos \left(\alpha_{n}\right)\right) \\
& Q_{c h}(t)=\sum_{n=1}^{8} A_{n} \sin \left(\theta_{n}+2 \pi f_{d} t \cos \left(\alpha_{n}\right)\right)
\end{aligned}
$$


where $A_{n}$ represents the amplitude, $\alpha_{n}$ the arrival angle and $\theta_{n}$ the phase of $n$-th ray. $f_{d}$ represents the maximum Doppler spread. $\alpha_{n}$ denotes the azimuth arrival angle for the $n$-th path and is defined as:

$$
\alpha_{n}=\frac{\pi}{4}(n-1) \text { where } n=1, \ldots, 8
$$

$\theta_{n}$ represents the initial phase of each path defined as:

$$
\theta_{n}=2 \pi U
$$

where $U$ is a uniformly distributed random variable whose values lie between 0 and 1 .

As shown in Figures $5 \mathrm{a} \& 5 \mathrm{~b}$, the presence of Doppler can result in an approximate $1 \mathrm{~dB}$ degredation in $\mathrm{S} / \mathrm{N}$ performance. The pilot correction process prevents the generation of an irreducible error floor. The performance of the modem is virtually unaffected at low Doppler spreads. It can be seen that a Signal to Noise Ratio (SNR) of $32 \mathrm{~dB}$ per bit is required to achieve an uncoded Bit Error Rate (BER) of 1 in 1000 bits.

The modem design presented here is virtually transparent to IF frequency offsets up to $\pm 150 \mathrm{~Hz}$ and Doppler spreads of $\pm 25 \mathrm{~Hz}$. A further increase in these values could be achoeve but would require the gap bandwidth and tone extraction filters to be redesigned. Large values of gap bandwidth allow more noise to enter the tone extraction filters and thus produce noisier references. This degrades system performance.

In a simulation, it is mathematically intractable to include the effects of channel fading after the transmit IF processing. Since modulation is a linear process, the channel fading can be simulated at baseband prior to IF modulation. While this approach is physically unrealistic, it does not effect system performance.

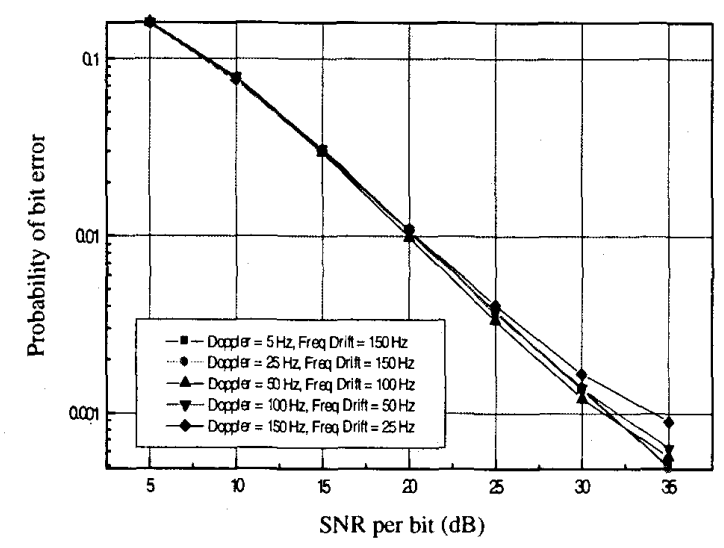

Figure 5a: BER of 32-QAM in Rayleigh fading for different values of Doppler spread and frequency offsets $(R=0.2)$

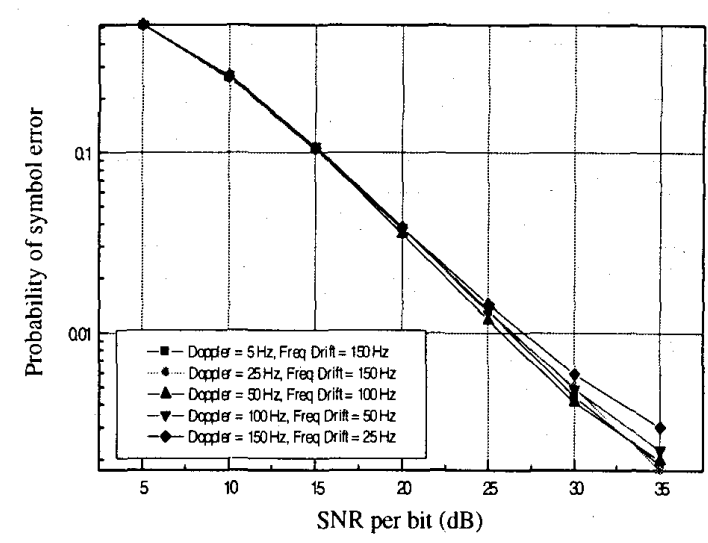

Figure 5b: SER of 32-QAM in Rayleigh fading for different values of Doppler spread and frequency offsets $(R=0.2)$

\section{PERFORMANCE IN RICIAN FADING}

Many channels have a dominant component and are more accurately characterised using Rician fading statistics. The effect of the Rician K-factor has been evaluated by simulating the modem in a Rician environment.

Rician fading is generated by extending the Rayleigh fading model to include a single dominant multipath. The ratio of the direct or dominant power $P_{d}$ to the indirect or scattered power $P_{i d}$ is simulated by defining the Rician K-factor:

$$
K=P_{d} / P_{i d}
$$

In practice, this value is often quoted in $\mathrm{dB}$ as $10 \log K$. It is convenient in simulations to assume that the dominant path arrives at an azimuth angle of zero. This results in a slightly modified In-phase component. The In-phase and Quadrature components for a Rician channel are defined as below:

$$
\begin{gathered}
I_{R c h}(t)=\sqrt{K \sum_{n=1}^{8} A_{n}^{2}}+\sum_{n=1}^{8} A_{n} \cos \left(\theta_{n}+2 \pi f_{d} t \cos \left(\alpha_{n}\right)\right) \\
Q_{R c h}(t)=\sum_{n=1}^{8} A_{n} \sin \left(\theta_{n}+2 \pi f_{d} t \cos \left(\alpha_{n}\right)\right)
\end{gathered}
$$

Figures $6 \mathrm{a}$ and $6 \mathrm{~b}$ indicate that for low $\mathrm{K}$-factors the performance is close to that of a Rayleigh channel. However, as the value for the K-factor increases, the performance tends towards the AWGN case. For fixed links, the use of directional antennas will tend to result in more Rician channels and therefore an improved performance versus signal to noise. 


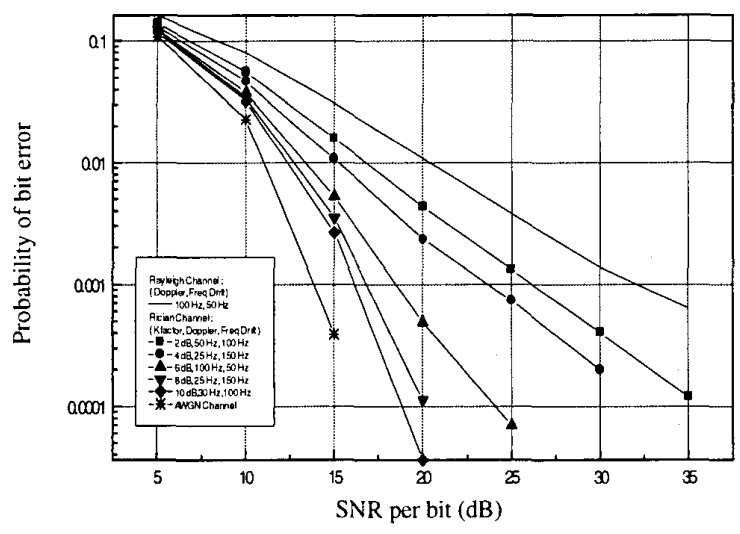

Figure 6a: BER of 32 QAM in Rician channel for different valuse of $K$ factor, Doppler spread and frequency offsets

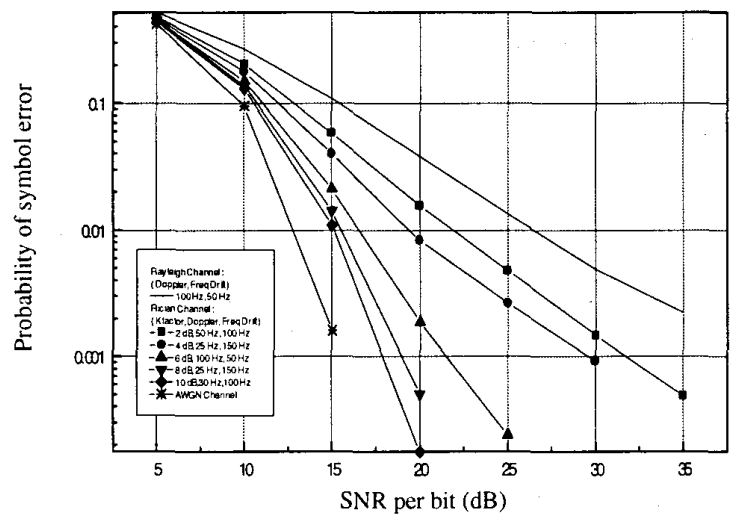

Figure 6b: SER of 32 QAM in Rician channel for different valuse of $K$ factor, Doppler spread and frequency offsets

\section{CONCLUSIONS}

In this paper a pilot tone aided 32 QAM modem has been developed offering a basic $64 \mathrm{kbit} / \mathrm{s}$ ISDN service (70 $\mathrm{kbit} / \mathrm{s}$ gross) in a $20 \mathrm{kHz}$ channel. A transceiver design has been produced that automatically corrects for the effects of multiplicative channel fading and local oscillator frequency drifts. The architecture supports simple pilot tone correction using a novel process of pilot detection and pre-distorted subband down-conversion. The design avoids the need for Hilbert transforms in the data extraction path.

The simulation of the 32 QAM PTA modem in AWGN indicates an optimum pilot tone to data power ratio of 0.2 . Statistical analysis of the transmitted signal power has shown that the transmit envelope remains below a value of $9.24 \mathrm{~dB}$ (relative to the mean) for $99.9 \%$ of the time.

A SNR of $32 \mathrm{~dB}$ is required to achieve a BER of $10^{-3}$ in worst case Rayleigh fading conditions. Transmission through Rician fading improves the required SNR and for the highest values of $\mathrm{K}$ factor (i.e a Gaussian channel) the required SNR approaches $14 \mathrm{~dB}$ for a BER of $10^{-3}$.

The use of pilot tones makes 32 QAM virtually transparent to errors introduced by Doppler and frequency offsets. Given the rigorous performance of the modem in Rayleigh and Rician channel, the design is expected to be suitable for both fixed wireless and mobile radio applications.

\section{ACKNOWLEDGEMENTS}

The authors would like to acknowledge Professor Joe McGeehan for his help during the modem development and for the provision of original literature relating to the process of pilot tone correction. This work was funded by Linear Modulation Technology.

\section{REFERENCES}

[1] J G Proakis, Digital Communications, McGraw Hill, $3^{\text {rd }}$ Edition, 1995

[2] Sampei S \& Sunaga T, Rayleigh Fading Compensation for QAM in. Land Mobile Radio Communication, IEEE Transactions on Vehicular Technology, Vol. 42, No. 2, May 1993, pp. 137-147.

[3] J.P.McGeehan and A. Bateman, Phase Locked Transparent Tone-In-Band (TTIB): A New Spectrum Configuration Particularly Suited to the Transmission of Data Over SSB Mobile Radio Networks, IEEE Trans. Commun., Vol.COM-32, pp81-87, Jan. 1984.

[4] Cavers J K, Liao M, A Comparison of Pilot Tone \& Pilot Symbol Techniques for Digital Mobile Communications, Globecom 92, 1992, pp. 915-921.

[5] Martin P M, Bateman A, McGeehan J P, Marvill JD, Implementation of a 16 QAM data system using TTIB-based fading correction techniques, IEEE Vehicular Technology Conference, 1988, pp. 71-76.

[6] Bateman A, Feedforward transparent tone-in-band: Its implementations and applications, IEEE Transactions on Vehicular Technology, Aug 1990, Vol.39, No.3, pp.235-243

[7] A.R. Nix, A Fundamental Investigation into Short Range High Capacity Mobile Data Transmission, University of Bristol PhD Thesis, September 1993. 